\title{
Vector-Based Analysis of the Similarity Between Breathing and Heart Rate During Paced Deep Breathing
}

\author{
Denis Kleyko ${ }^{1}$, Evgeny Osipov ${ }^{1}$, Urban Wiklund ${ }^{2}$ \\ ${ }^{1}$ Luleå University of Technology, Luleå, Sweden \\ ${ }^{2}$ Umeå University, Umeå, Sweden
}

\begin{abstract}
The heart rate (HR) response to paced deep breathing $(D B)$ is a common test of autonomic function, where the scoring is based on indices reflecting the overall heart rate variability $(H R V)$, where high scores are considered as normal findings but can also reflect arrhythmias. This study presents a method based on hyperdimensional computing for assessment of the similarity between feature vectors derived from the HR and breathing signals. The proposed method was used to identify subjects where HR did not follow the paced breathing pattern in recordings from $D B$ tests in 174 healthy subjects and 135 patients with cardiac autonomic neuropathy. Subjects were classified in 4 similarity classes, where the lowest similiarity class included 35 patients and 3 controls. In general, the autonomic function cannot be evaluated in subjects in the lowest similarity class if they also present with high HRV scores, since this combination is a strong indicator of the presence of arrhythmias. Thus, the proposed vector-based similarity analysis is one tool to identify subjects with high $H R V$ but low cardiorespiratory synchronization during the $D B$ test, which falsely can be interpreted as normal autonomic function.
\end{abstract}

\section{Introduction}

This paper explores principles of hyperdimensional computing for similarity analysis of heart rate variability (HRV) and respiration (RESP) signals during the deep breathing (DB) test. The test is rather simple: during one minute, a subject takes six paced deep breaths while HRV and often RESP signals are recorded. The autonomic function is normally scored using the total power of recorded HRV signal or by the deep breathing index (DBI), which is calculated as the average of the heart rate difference in each breathing cycle. It is important to note that these indices assume that RESP and HRV signals are synchronized with each other, since the actual pattern of the heart rate fluctuations is ignored. This assumption may cause issues: for ex- ample, if there are subjects presenting with subtle arrhythmias during the DB test. In such cases, the DB test is not an adequate test for evaluating autonomic function. In order to identify such subjects, we propose to explore methods capable of analyzing cardiorespiratory synchronization. In particular, the paper studies the similarity between HRV and RESP signals from DB tests using feature-based analysis, where feature vectors (patterns) are mapped to hyperdimensional space (HD space) [1]. Computing in HD space operates with vectors of very high dimension and allows a computationally efficient analysis of complex dependencies between a large number of features. Patterns in HD space are then classified into different regions of cardiorespiratory synchronization/desynchronization using kmeans clustering.

\section{Recordings from patients and controls}

HRV (derived from a single-channel ECG) and RESP signals were recorded during DB tests ( 6 breaths/min during one min, corresponding to $0.1 \mathrm{~Hz}$ ) in the supine position. Data consisted of 174 healthy controls (53\% men, mean age 49 years, range 22-84 years) and in 135 patients $(51 \%$ men, mean age 51 years, range $26-76$ years) with different degrees of cardiac autonomic dysfunction (CAN) and arrhythmias. The CAN group consisted of patients with the disease transthyterin amyloidosis. These patients were selected since they often present with reduced HRV due to cardiac autonomic dysfunction, but falsely increased HRV due to subtle cardiac arrhythmias are also common in these patients [2].

\section{Methods}

Heart beats were detected in the recorded ECG:s (sampled at $500 \mathrm{~Hz}$ ) using a threshold-based algorithm. Spurious ectopic beats were removed by interpolation, but recordings with frequent ectopic beats were not edited, which was the case in less than 10 patients. HRV signals were constructed from RR intervals by cubic spline interpolation and resampling at $2 \mathrm{~Hz}$. The overall response 
in heart rate was quantified by the commonly used deep breathing index (DBI), calculated as the average of the heart rate difference in each breathing cycle. DBI was ageadjusted based on linear regression analysis of logarithmically transformed data from controls, from which Z-scores were determined as previously described in [3].

Fourier series (FS) modeling was used to analyze the characteristics of the HRV and RESP signals [3]. The DB test was performed with six cycles of paced breathing during one minute, corresponding to a breathing frequency, $f_{\text {resp }}=0.1 \mathrm{~Hz}$. By assuming that the complete one-minute test is periodic, each signal can be modelled as

$$
x(n)=\sum_{k=0}^{N-1} c_{k} e^{j 2 \pi T k n / N},
$$

where $T$ is the sampling interval, and $c_{k}$ is the amplitude and $N$ is the number of frequency components, which was was set to $N=18$. The power of each signal component is given by $P_{k}=\left|c_{k}\right|^{2}$. Note that the fundamental frequency, i.e., the frequency of the first component, is equal to the inverse of the period length (which is one minute). Thus, a signal with a nearly identical response for all six breathing cycles will present with most power at the breathing frequency and its harmonics, i.e., mainly at the $6^{t h}, 12^{t h}$ and $18^{\text {th }}$ components in the FS model. However, signals with irregular responses will also have power in other FS components, such as arrhythmias but also amplitude or frequency modulated signals.

The main aim of the vector-based similarity analysis is to detect HR responses that were unsynchronized with respiration. Therefore, three characterizing feature variables were derived based on spectral regions where the power was expected to be low if HR followed the paced breathing pattern, provided that the DB test was correctly performed. First, FS components were normalized based on the sum of the expected dominating components:

$$
c_{i}=P_{i} /\left(P_{6}+P_{12}+P_{18}\right) .
$$

Then a dimension reduction was performed by defining three feature variables, representing the power in different frequency regions:

$$
\begin{gathered}
x_{1}=c_{\text {lowfreq }}=\sum c_{i}, i=\{1-4\} \\
x_{2}=c_{\text {midfreq }}=\sum c_{i}, i=\{5,7\} \\
x_{3}=c_{\text {highfreq }}=\sum c_{i}, i=\{8-11,13-17\}
\end{gathered}
$$

These features were used to assess the similarity between the feature vectors representing the RESP and HRV signals and for classification of different patterns.

The feature vectors were mapped to HD space where the normalized Hamming distance (HamD) between the two HD vectors was used as the similarity metric. Table 1 provides and example of such mapping. First, a value of each normalized feature was quantized. The table shows the number of the corresponding quantization level. Next, each feature was randomly assigned a base binary HD vector with $N=10000$ dimensions ( $N=8$ in the table). Base HD vectors have approximately the same number of 1 's and 0's. Coded HD vectors corresponding to quantization levels were derived from the base HD vectors using the "nonlinear mapping" procedure as described in [4]. Qualitatively, the larger is the level number the more dissimilar is its coded HD vector to the base HD vector (red positions in the table). Finally, coded HD vectors for all features were summed together (bitwise) in a single HD vector (HD pattern) which was binarized using the majority sum operation.

Table 1. Example of the mapping of a feature vector to HD space.

\begin{tabular}{|c|c|c|c|}
\hline Feature & $\mathrm{X} 1$ & $\mathrm{X} 2$ & $\mathrm{X} 3$ \\
\hline Normalized & 0.25 & 0.3 & 0.02 \\
\hline Quantized & 5 & 6 & 1 \\
\hline Base vector & 00110100 & 11100110 & 01000110 \\
\hline Coded vector & 01010110 & 10001101 & 01010100 \\
\hline HD pattern & \multicolumn{3}{|c|}{01010100} \\
\hline
\end{tabular}

The similarity between two binary HD vectors, $\mathbf{X}_{r}$ and $\mathbf{X}_{h}$, is characterized by the Hamming distance (HamD), which is normalized by the dimensionality $N$ ) and measures the proportion of elements in which they differ and is calculated as:

$$
\operatorname{HamD}\left(\mathbf{X}_{r}, \mathbf{X}_{h}\right)=\frac{1}{N} \sum_{i=1}^{N}\left(X_{r i} \oplus X_{h i}\right),
$$

where $\oplus$ denotes element-wise XOR operation. The similarity of the signals (HD patterns) was classified into four different groups based on k-means clustering $(k=4)$ of normalized HamD.

Power spectra of linearly detrended and Hanningwindowed data were determined using the Welch periodogram method, and power spectral percentiles were determined for each group. A comparison was also made with a coherence-based similarity index, the coherent power index (CPI), given by:

$$
C P I=\int_{f=0}^{f_{s} / 2} p_{1}(f) c_{12}(f) d f / \int_{f=0}^{f_{s} / 2} p_{1}(f) d f
$$

where $p_{1}(f)$ is the power spectrum of HRV and $c_{12}(f)$ is the coherence spectrum. Thus, CPI is an estimate of the fraction of the total power of the HRV signal which is coherent with the RESP signal. Coherence spectra were determined by dividing data into 20 seconds blocks, with 10 seconds overlap between blocks. 


\section{4. $\quad$ Results}

Figure 1 shows two examples of recorded HR signals and how feature variables were derived from FS components. Left panels shows data from a healthy subject with a relatively high similarity between HRV and RESP, as shown by the FS components. Right panels shows data from a patient with dysrhythmia, where there was a large difference in the pattern of FS components for HRV as compared to RESP. This patient presented with seconddegree atrioventricular block in three cycles, thus this is an example of marked cardiorespiratory desynchronization.
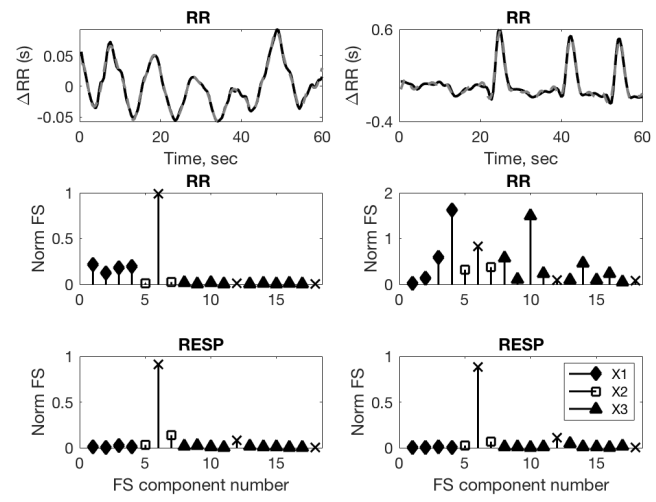

Figure 1. Recording from a healthy subject (left) and from a patient with dysrhythmia (right). Top: Original RR signals and the reconstructed ones from 18 FS components. Normalized FS coefficients for HRV (middle) and RESP (bottom) signals. Symbols show how FS components were merged into three feature variables.

Figure 2 presents the distribution of the three extracted features from FS components of the recorded RESP (top) and HRV (bottom) signals for Controls (left) and CAN patients (right). Box plots of features for RESP signals are similar for both groups, while values of features for HRV signals are lower for controls than that for CAN patients.

Figure 3 shows a comparison of the two similarity measures for the recorded data. For each pair of RESP and HRV signals, CPI and HamD were calculated. The figure demonstrates that there is an interrelation between HamD and CPI. Pairs with low CPI feature high HamD while pairs with high CPI correspond to low HamD. Note, that there are exceptions when large CPI corresponds to rather large HamD. It is also clear in Figure 3 that most of the controls reside in the upper left corner, which corresponds to the area with large CPI and small HamD. Another observation is the majority of the points in the lower right corner (i.e., the region with low similarity) are from CAN patients.

Four different groups obtained after k-means clustering
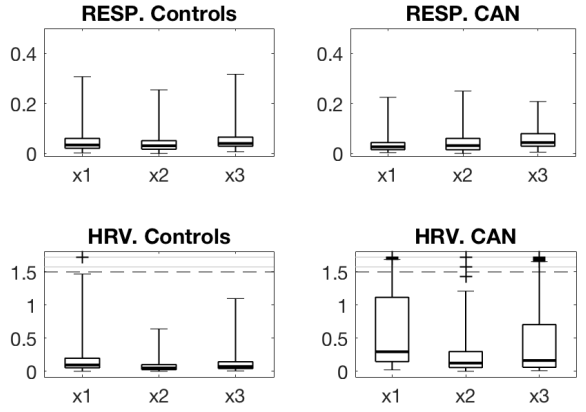

Figure 2. Box plots illustrating distributions of features calculated from normalized FS for each category of subjects in the recorded data. Boxes show median and interquartile ranges. Dashed lines indicate the threshold for defining extreme values, which are shown in a compressed region between the solid lines.

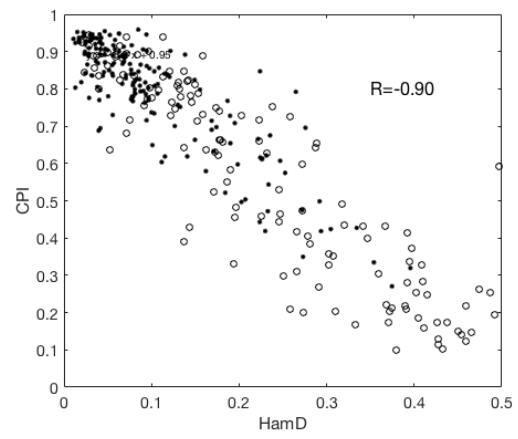

Figure 3. CPI against HamD for controls $(\bullet)$ and patients (o).

Table 2. Classification of subjects into similarity classes based on k-means clustering of HamD and CPI.

\begin{tabular}{lcr}
\hline \hline HamD similarity & Controls & CAN patients \\
\hline Very high & $120(69 \%)$ & $33(24 \%)$ \\
High & $30(17 \%)$ & $39(29 \%)$ \\
Moderate & $21(12 \%)$ & $28(21 \%)$ \\
Low & $3(2 \%)$ & $35(26 \%)$ \\
\hline \hline CPI similarity & Controls & CAN patients \\
\hline Very high & $115(66 \%)$ & $40(30 \%)$ \\
High & $43(25 \%)$ & $34(25 \%)$ \\
Moderate & $14(8 \%)$ & $27(20 \%)$ \\
Low & $2(1 \%)$ & $34(25 \%)$ \\
\hline \hline
\end{tabular}

could be naturally associated with the degree of similarity between HRV and RESP signals. Class $\mathrm{C} 1$ denotes very high similarity class, C2 - high similarity, C3 - moderate similarity, and $\mathrm{C} 4$ - low similarity. Table 2 shows the distribution of signals into different classes for HamD and 

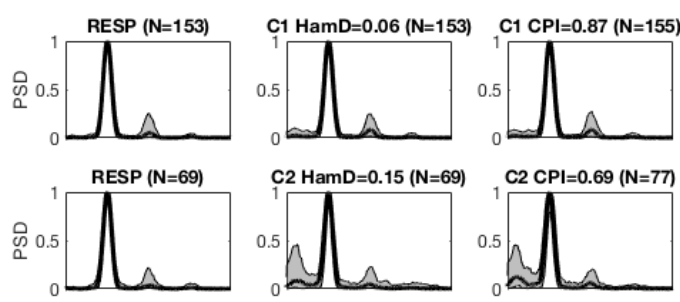

C2 $\mathrm{HamD}=0.15(\mathrm{~N}=69)$
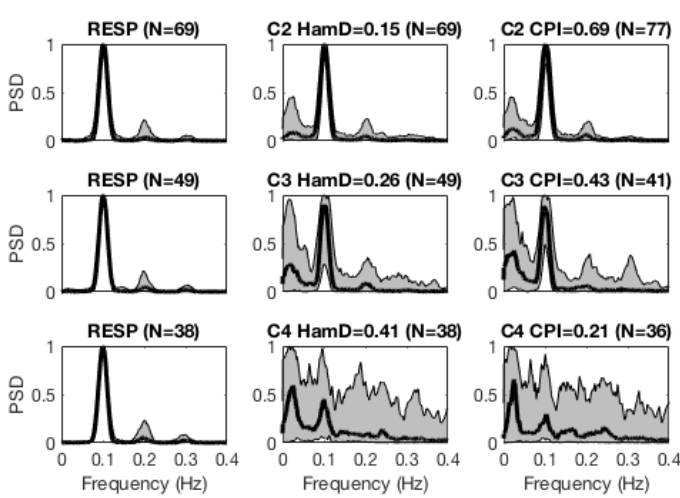

Figure 4. Power spectra for respiration signals (left) and for heart rate signals (middle) after classification of the HamD into four classes. Right panels show corresponding power spectra for heart rate after classification of coherent power index (CPI) into four classes. Lines show medians and areas illustrate $10 \%$ and $90 \%$ percentiles for normalized power spectral densities (PSD) for all signals in each class.

CPI respectively. The distributions obtained for both metrics are very similar. Notably, there was a high percentage ( $26 \%$ for HamD) of CAN patients classified into low similarity class while there were only $2 \%$ of Controls in that class. This is a clear indication of the presence of low cardiorespiratory synchronization in many CAN patients.

Figure 4 shows a comparison between spectral percentiles for HamD and CPI after the clustering. The spectral percentiles showed a clear distinction between different degrees of HRV responses, ranging from highly similar to RESP, with a marked peak at $0.1 \mathrm{~Hz}$ and its harmonics in the highest similarity class $(\mathrm{C} 1)$, to broadband spectral patterns in the lowest similarity class (C4).

Figure 5 depicts HamD against DBI for both groups of subjects, where the interpretation of DBI in terms of autonomic function is marked in different regions. The upper right corner indicates the region with both high DBI and high HamD, indicating low synchronization between RESP and HRV signals due to arrhtyhmias. Note that there were 16 CAN patients but no Controls in that region. These patients should be excluded from the evaluation of autonomic function. On the other hand, a z-score for DBI below -2 most likely indicates reduced autonomic function event if HamD is high, since the magnitude of the HR fluctuations is so low.

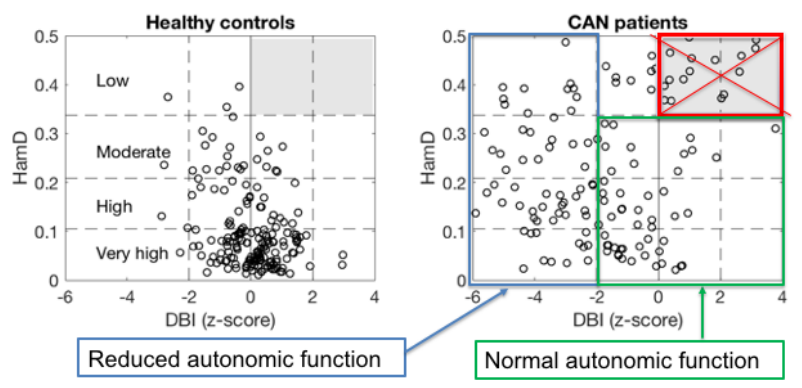

Figure 5. HamD between RESP and HRV signals against DBI (z-score) for controls and CAN patients. Horizontal lines indicate boundaries for similarity classes found using k-means clustering. Boxes indicate regions with normal DBI, reduced DBI, and exclusion from scoring of autonomic function.

\section{Conclusion}

The proposed vector-based similarity analysis is advantageous as it can be based on all types of feature variables, and also presented with equivalent results as the coherence-based analysis. The similarity analysis helped to identify and exclude subjects with HR responses due to arrhythmia during the deep breathing test, where scores as DBI then can be used to score autonomic function in the remaining subjects.

\section{References}

[1] Kanerva P. Hyperdimensional computing: An introduction to computing in distributed representation with highdimensional random vectors. Cognitive Computation 2009; 1(2):139-159.

[2] Wiklund U, Hornsten R, Karlsson M, Suhr OB, Jensen SM. Abnormal heart rate variability and subtle atrial arrhythmia in patients with familial amyloidotic polyneuropathy. Ann Noninv Electrocard 2008;13(3):249-256.

[3] Wiklund U, Kadkhodaee A, Andersson K, Suhr OB, Hörnsten R. Normal scores of deep breathing tests: beware of dysrhythmia in transthyretin amyloidosis. Amyloid 2018; 25:54-61.

[4] Kleyko D, Rahimi A, Rachkovskij D, Osipov E, Rabaey J. Classification and Recall with Binary Hyperdimensional Computing: Trade-offs in Choice of Density and Mapping Characteristic. IEEE Transactions on Neural Networks and Learning Systems 2018;PP(99):1-19.

Address for correspondence:

Name: Urban Wiklund

Full postal address: SE-901 87 Sweden, Umeå, Umeå University, Department of Radiation Sciences, Biomedical Engineering.

E-mail address: Urban.Wiklund@umu.se 